
\title{
3 Research Square \\ SLC40A1 mediates ferroptosis and cognitive dysfunction in type 1 diabetes
}

\author{
Lijun Hao \\ Shanxi Provincial People's Hospital \\ Jun Mi \\ Shanxi Provincial People's Hospital \\ Liping Song \\ Shanxi Provincial People's Hospital \\ Yinnan Guo \\ Shanxi Provincial People's Hospital \\ Yanli Li \\ Shanxi Medical University \\ Yiru Yin \\ Shanxi Medical University \\ Ce Zhang ( $\square$ doc_zhangce@yeah.net ) \\ Shanxi Medical University https://orcid.org/0000-0003-4068-9626
}

\section{Research}

Keywords: Type 1 diabetes, cognitive dysfunction, resting state functional magnetic resonance imaging, mRNA/LncRNA array, Slc40a1, ferroptosis

Posted Date: June 22nd, 2020

DOl: https://doi.org/10.21203/rs.3.rs-29323/v2

License: (c) (1) This work is licensed under a Creative Commons Attribution 4.0 International License. Read Full License

Version of Record: A version of this preprint was published at Neuroscience on May 1st, 2021. See the published version at https://doi.org/10.1016/j.neuroscience.2021.03.009. 


\section{Abstract}

Objective: Cognitive dysfunction often accompanies diabetes. Both hypoglycemia and hyperglycemia cause cognitive dysfunctions. However, the underlying pathophysiology remains unclear. Recent evidence show that ferroptosis primarily triggers nerve cell death, Alzheimer's disease (AD), Huntington's disease $(H D)$, and Parkinson's disease (PD). We purposed to investigate whether ferroptosis is a vital pathogenic pathway in diabetes-induced cognitive dysfunction.

Methods and results: Type 1 diabetic rat model was created by intraperitoneal injection of streptozotocin (STZ). Significant cognitive dysfunction was observed in the diabetic rats as evidenced by increase in latency period to find a hidden platform and decreased cumulative time spent in the target quadrant in the Morris water maze test. We detected the amplitude of low-frequency fluctuation (ALFF) of the BOLD (Blood Oxygenation Level-Dependent) signal using resting-state functional magnetic resonance imaging (rs-fMRI). Consequently, we found that the ALFF values, as well as the T2 relaxation time of the bilateral hippocampus, were reduced in Type 1 diabetic rats. We detected $\mathrm{Fe}^{2+}$ level and lipid peroxidation products (MDA and 4-HNE) in the hippocampus. Mitochondria and neuron injury in the STZ-induced diabetic rats were determined using a Transmission Electron Microscope and Nissl body staining. Iron overload and ferroptosis were detected in the hippocampus. Furthermore, mRNA microarray analysis revealed 201 dysregulated mRNAs in STZ-induced type 1 diabetes (T1D). Pathway enrichment analyses indicated that differentially expressed mRNAs associated-coding genes were associated with ferroptosis. Among ferroptosis signaling pathway genes, Slc40a1 gene (ferroportin) was downregulated.

Conclusion: We show that ferroptosis is associated with diabetic cognitive dysfunction and Slc40a1 mediates ferroptosis in Type 1 diabetes.

\section{Introduction}

Diabetes (diabetes mellitus, DM) is a metabolic disease characterized by hyperglycemia. It has been increasing in recent years and has become a global public health issue. Globally, it is estimated that the number of adult diabetes patients will reach 642 million by 2040 [1]. Diabetes often results in many chronic complications, including cardiovascular disease, kidney disease, retinopathy, peripheral neuropathy, and brain complications [2]. Due to its high morbidity, long course of the disease, and many complications, DM is a threat to human health with a substantial economic burden on families and society [3]. Therefore, for considerable social significance, it is necessary to conduct research on the prevention and treatment of diabetes and its complications.

Among the DM complications, there is limited research on cognitive dysfunctions. Some patients with type 1 and 2 DM show cognitive dysfunctions with a remarkable negative impact on their daily living activities. Both hypoglycemia and hyperglycemia have been shown to cause cognitive dysfunctions. The pathophysiology of cognitive dysfunction DM patients include: (1) the role of hyperglycemia, (2) the role of the vascular disease, (3) the role of hypoglycemia, and (4) the role of insulin resistance and amyloid 
[4]. However, the underlying pathophysiology remains unclear [5]. In 2012, the Cell Death Nomenclature Committee (NCCD) proposed a new mechanism of programmed cell death (Ferroptosis) [6]. This mechanism is iron-dependent fatal lipid peroxidation induced cell death and the process is characterized by an increase in intracellular iron, and lipid peroxidation [7]. Morphologically, the mitochondria shrink, its membrane thickens, and the ridges decrease or disappear [8]. Henceforth, internationally, ferroptosis has attracted much research attention. A significant number of animal model studies have shown that the iron overload causes accumulation of iron in the brain, resulting in severe cognitive deficits $[9,10]$ and is a crucial injury mechanism in neurodegenerative diseases [11]. Currently, it is believed that ferroptosis is the main trigger of nerve cell death [12], including Alzheimer's disease (AD) [13], Huntington's disease (HD) [14], and Parkinson's disease (PD) [15]. Moreover, AD has been referred to as diabetes of the brain or type 3 diabetes [16]. We, therefore, hypothesized that iron deposition and ferroptosis constitutes an important pathogenic pathway in Diabetes-induced cognitive dysfunction.

We first established a Streptozotocin (STZ)-induced T1D rat model. After that, we investigated the behavioral impact and cerebral alterations in the model through behavioral phenotyping and resting-state functional magnetic resonance imaging ( $r$-fMRI). We further examined the iron-ion $\left(\mathrm{Fe}^{2+}\right)$ level and products of lipid peroxidation (MDA and 4-HNE) in the hippocampus. Mitochondria were also observed through a transmission electron microscope. Finally, the expression profiles of mRNA/IncRNA were assayed using a rat microarray to examine ferroptosis signaling. Herein, we aimed to assess whether ferroptosis contributes to the pathogenesis of diabetes-induced injury to neurons; therefore provides potential therapeutic targets.

\section{Materials And Methods}

\section{Rats and diabetic model}

The Institutional Animal Care and Use Committees approved the rats' experimental protocols. All the experimental protocols were strictly performed as per the national guidelines for the care and use of experimental animals. Sixty mature male Sprague-Dawley (SD) rats (weight $=250 \sim 270 \mathrm{~g}$ ) were obtained from SPF Biotechnology Co., LTD (Beijing). We housed the rats in cages in groups of three and maintained under standard conditions $\left(20^{\circ} \mathrm{C} \sim 25^{\circ} \mathrm{C}, 50 \% \sim 65 \%\right.$ relative humidity, $12 \mathrm{~h}$ light: $12 \mathrm{~h}$ dark light cycle) with ad libitum access to rodent pellet diet and water. After a 7-day acclimatization period, we randomly assigned the rats into the control group $(n=20)$ and the model group $(n=40)$. The rats in the model group were subjected to $12 \mathrm{~h}$-fasting and then subjected to the regime of STZ-induced T1D. Briefly, the rats were intraperitoneally (i.p.) injected with $55 \mathrm{mg} / \mathrm{kg} \mathrm{STZ}$ (diluted in $0.01 \mathrm{M}$ sodium citrate, $\mathrm{pH}$ 4.5; Sigma-Aldrich, St. Louis, MO, USA. S0130-500MG). The rats in the control arm received an injection of $0.01 \mathrm{M}$ sodium citrate. We measured blood glucose levels (by the tail) daily using a glucose meter. The rats in the model arm with blood glucose level $\geq 16.6 \mathrm{mmol} / \mathrm{I}$ at three days post-STZ-injection were included in our study. All rats were sacrificed at eight weeks post-STZ injection.

\section{Morris water maze}


Body weights and fasting blood glucose levels (by the tail) were monitored weekly. After eight weeks of modeling, we used the Morris water maze for behavioral testing. The water maze experimental device constituted a cylindrical pool with a diameter of $150 \mathrm{~cm}$ and a height of $60 \mathrm{~cm}$, which we artificially divided into four quadrants. During the experiment, we filled the pool with water to a depth of $30 \mathrm{~cm}$. The temperature was controlled at $23 \pm 2^{\circ} \mathrm{C}$. A circular platform $(10 \mathrm{~cm} \times 10 \mathrm{~cm})$ was placed about $1 \mathrm{~cm}$ below the level of the target quadrant (TQ). The pool was surrounded by curtains to avoid interference from the external environment. Various striking visual stimulus pictures were posted on the wall of the pool to help rats learn to identify the direction. Briefly, we individually placed the rats in the apparatus in one of the four different quadrants and allowed them up to 60 s to reach the submerged platform in the hidden platform test. We guided the rats to the submerged platform if they failed to find it, and allowed all of them to remain on the platform for an additional 10s. The behavioral data were recorded automatically and analyzed using a video tracking system (\#WMT-100S, Techman soft, Chengdu, Sichuan, China). We performed an hidden platform test for five days, and on the 6th day, the probe trial test was conducted without a hidden platform. During the test, the cumulative time spent in the target quadrant was recorded and analyzed using the same video computer system.

\section{Total RNA preparation and LncRNA microarray analysis}

The total RNA was isolated from the hippocampus region. We used $1 \%$ agarose gel electrophoresis and NanoDrop ND-1000 (Thermo Fisher Scientific, Waltham, MA, USA) for sample quantification and qualification. Subsequently, we amplified and transcribed the total RNA into fluorescent cRNA using the Agilent Array platform, followed by hybridization using the Rat LncRNA Microarray v2.0 (Arraystar, Rockville, USA, including 13,611 IncRNAs and 24,626 protein-coding mRNAs) overnight. After that, the microarray was scanned using an Agilent DNA Microarray ScannerG2505C (Agilent Technologies, Santa Clara, CA, USA). We analyzed and processed the acquired images using the Agilent Feature Extraction (v11) and GeneSpringGXv12.1 (Agilent Technologies). Standard routine analyses, including probe summarization (RMA), quality control, and probe annotation, were performed. The differentially expressed IncRNA (DE IncRNAs) and genes (DEGs) between groups were identified using the $t$-test with the criteria of fold change $>1.5$ and $p<0.05$.

\section{Gene Ontology (GO) and KEGG analysis}

The Gene Ontology (GO) categories of DEGs were derived from the GO database (http://www.geneontology.org). We determined the KEGG pathways associated with DEGs based on the (Kyoto Encyclopedia of Genes and Genomes, http://www.genome.jp/kegg). KEGG pathways and GO terms with $p<.05$ were considered as categories of interest.

\section{Quantitative real-time PCR (qRT-PCR)}

The relative expression levels of four target mRNAs (including SLC40A1) and IncRNAs in the rat brain were determined using qRT-PCR using the primers listed in Table 1. Briefly, we extracted total RNA from the rat hippocampus using the TRIzol reagent (TaKaRa, Tokyo, Japan) as per the manufacturer's 
protocol. After that, we synthesized first-strand CDNA and amplified following the procedure: denaturation at $94^{\circ} \mathrm{C}$ for $2 \mathrm{~min} ; 40$ cycles of $94^{\circ} \mathrm{C}$ for $20 \mathrm{~s}, 58^{\circ} \mathrm{C}$ for $20 \mathrm{~s}, 72^{\circ} \mathrm{C}$ for $20 \mathrm{~s}$; and a final extension at $72^{\circ} \mathrm{C}$ for $4 \mathrm{~min}$. The amplification was performed on an Agilent StratageneMx3000PRT-PCR machine (Agilent Technologies) using a Bestar ${ }^{\circledR}$ Sybr Green qPCR master mix kit (DBI Bioscience, Shanghai, China). We used GAPDH as the internal reference gene in our study. The relative expression levels of target mRNAs and IncRNAs were computed using the $2^{-\Delta \Delta C t}$ methods.

\section{Western blot analysis}

We utilized Western blotting analyses to examine the expression of SLC40A1 protein in the rats' brains. The protein lysates were extracted from the rats' hippocampus using the lysis buffer (Beyotime). We used the Bradford protein assay kit (Thermo Fisher Scientific Inc.) to quantify the protein samples following the manufacturer's protocol, followed with separation using 10\% SDS-PAGE. We performed protein immunoblotting on the PVDF membrane (Merck Millipore, Billerica, MA, USA) with primary antibodies against SLC40A1 (1: 500 ab58695区Abcam, Cambridge, UK) and GAPDH (1: 10000, Abcam). HRP goat anti-rabbit/mouse IgG was used as the secondary antibody (1: 20000, Boster Biotechnology). The membranes were managed with enhanced chemiluminescence $(E C L)$ and then examined using imagePro Plus 6.0 software. The densitometric data were normalized to the GAPDH level.

\section{Resting state functional magnetic resonance imaging (rs-fMRI) and relaxation time test}

We placed the rats in a special anesthesia box and anesthetized them with $2.5 \%$ isoflurane mixed with oxygen, and then fixed the prone position on the rat's special scan bed. The head of the rat was fixed with a dental rod and an ear rod to prevent head movement during the scan. The rats' breathing, heart rate, temperature, and other vital signs were closely monitored, and the concentration of the inhaled anesthetics was adjusted in time to prevent accidental anesthesia during the entire data collection process. We utilized the water circulation heating system to maintain the rats' body temperature at about $37^{\circ} \mathrm{C}$. According to the depth of anesthesia, the breathing rate was maintained at about 45 times/minute.

We acquired the functional image using the Bruker's 7.0 T magnetic resonance imaging system (Pharmascan). The surface coil with a diameter of $38 \mathrm{~mm}$ was used to transmit the RF pulse signal and the four-channel surface coil to receive the signal. Repetition time $(T R)=2000 \mathrm{~ms}$, echo time $(T E)=20 \mathrm{~ms}$, field of view $=35 \mathrm{~mm} \times 35 \mathrm{~mm}$, layer thickness (thickness) $=0.55 \mathrm{~mm}$, flip angle (flip angle) $=90^{\circ}$, matrix size $=64 \times 64,600$ repetition. All original data were converted to DICOM format data using the Paravision 6.0 software that comes with the machine. The transverse relaxation time T2 (T2 relaxation time) uses a multi-layer multi-echo scanning sequence T2map-MSME. The main parameters of the sweep were as follows: (repetition time $(T R)=2855 \mathrm{~ms}$, slices (slices) $=12$, layer thickness (slice thickness) $=1.2 \mathrm{~mm}$, matrix size $=256 \times 256$, the field of view $=35 \mathrm{~mm} \times 35 \mathrm{~mm}$, echo spacing: $9 \mathrm{~ms}, \mathrm{TE}=9 / 18 / 27 /$ 36/45/54/63/72/81/90/99/108/117/126/135/144/153/162/171/180/189/198/207/126/225/ms, hand-drawn interested region (region of interest, ROI), T2 relaxation-time is calculated by workstation software Paravision6.0. The statistical parameter map toolkit (spmratIHEP) and the resting state 
functional magnetic resonance data processing assistant DPARSF software package were used for data analysis.

\section{Histopathological examinations}

We isolated the brain tissues and paraffin-embedded for Nissl's staining. Serial sections of $5 \mu \mathrm{m}$ were cut onto slides using a rotary microtome (Leica, Wetzlar, Germany) and deparaffinized. The protocol of staining methods was conducted using cresyl violet (Nissl's staining, Sigma-Aldrich) following the manufacture's instruction. We captured the morphological changes of the hippocampus area and hippocampal neurons using light microscopy (BX51, Olympus, Tokyo, Japan). We utilized ImageJ for digital pathology image analysis.

\section{Transmission electron microscope}

We used four specimens of the CA1 zone of the right hippocampus tissue about $1 \mathrm{~mm}^{3}$, and through fixation, rinsing, dehydration, infiltration, embedding, and other steps to make ultra-thin sections with a thickness of approximately $60-80 \mathrm{~nm}$. After that, we double-stained the specimens using uranyl acetate and lead citrate for $10 \mathrm{~min}$, respectively before overnight drying. We then selected some representative sections for observation using TEM (Hitachi H-7000FA. Wuhan University, China).

\section{Iron assay}

We estimated the Ferrous ion $\left(\mathrm{Fe}^{2+}\right)$ level in the hippocampus using the iron assay kit (MAK025, SigmaAldrich, USA) as per the manufacturer's instructions. Briefly, after washing the tissues in ice-cold PBS, we weighed and homogenized them in 6X volumes of iron assay buffer. After that, we added the iron reducer to each supernatant. The mixture was incubated for $30 \mathrm{~min}$, and the output was promptly assessed on a colorimetric microplate reader $(\mathrm{OD}=593 \mathrm{~nm})$. We normalized the $\mathrm{Fe}^{2+}$ level to the weight of the tissue samples.

\section{MDA assay}

We determined the content of MDA in the hippocampus using a Lipid Peroxidation (MDA) Colorimetric/Fluorometric Assay Kit『Catalog \# K739-100『BioVision『USA『as per the manufacturer's instructions. Briefly, we weighed the tissue samples and homogenized them in $30 \mathrm{X}$ volumes of buffer on ice. Subsequently, we centrifuged the tissue lysates at $13,000 \mathrm{~g}$ for $10 \mathrm{~min}$ at room temperature to collect the supernatant. The MDA in the hippocampus sample reacted with thiobarbituric acid (TBA) to generate an MDA-TBA adduct, which we calorimetrically quantified $(\mathrm{OD}=532 \mathrm{~nm})$. We normalized the MDA level to the weight of tissue samples.

\section{4-Hydroxynonenal (4-HNE) ELISA}

We used the 4-Hydroxynonenal (4-HNE) ELISA Kit (Catalog \# E4645-100, BioVision, USA) to determine the content of 4-HNE in the hippocampus according to the manufacturer's procedure. Briefly, we weighed and 
homogenized the tissue samples in $9 \mathrm{X}$ volumes of PBS. After that, we centrifuged the tissue lysates at $5,000 \mathrm{~g}$ for $10 \mathrm{~min}$ at room temperature to collect the supernatant and subsequently added the appropriate microtiter wells. We assayed all samples in triplicates and measured the optical absorbance at 450nm using a Synergy ${ }^{\mathrm{TM}}$ HT Multi-Mode Plate Reader (Biotek Instruments, Winooski, VT). A standard curve was constructed $(0-2000 \mathrm{pg} / \mathrm{ml})$, and then we determined the concentrations of 4-HNE in the hippocampus from the standard curve.

\section{Statistical analyses}

Data are expressed as mean \pm standard error (SEM). Utilizing Statistical SPSS 13.0 (GraphPad, USA) software, we performed the statistical analyses. The latency to the platform in the water maze was conducted using the two-way ANOVA. We used the Least Significant Difference Test (LSD-test) to compare the two groups. A comparison between the two groups was performed using the T-test, and $p$ $<.05$ considered as statistically significant. We constructed all the statistical graphs in GraphPad Prism 7.0 (GraphPad Software, Inc., Cary, NC).

\section{Results}

\section{STZ treatment successfully induced type 1 diabetic rat, and compromised learning and memory ability}

As shown in Figure 1, the blood glucose of rats increased sharply after intraperitoneal injection of STZ, and remained high for 8 weeks afterwards. Blood glucose levels remained normal in the control group ( $p$ $=0.00001$, Fig. 1A). The weight of rats decreased gradually along the disease time course. As the rats grow, their weight increased, which remained relatively stable with no obvious growth trend. The weight of rats in the control group increases over time. This caused a significant difference in weights between the two groups ( $p=0.012$, Fig. 1B). Further, it was observed that it took diabetic rats longer to find the hidden platform compared to the control rats $(p=0.004$, Fig. $1 C)$. In addition, the diabetic rats spent lower cumulative time in the target quadrant than rats of control group (Fig. 1D). Thus, we infer that STZ treatment effectively modeled diabetes in rats causing cognitive dysfunctions.

\section{The ALFF value and $\mathrm{T} 2$ relaxation time decreased in the brain of diabetic rats}

The rs-fMRI technology was employed to examine the changes in brain structure and function in diabetic rats. Notably, the ALFF value of the bilateral hippocampus and double cingulate cortex were lower in diabetic rats relative to control rats (Fig. 2A, blue areas). The transverse relaxation time $\mathrm{T} 2$ of the hippocampal region of interest was detected by magnetic resonance imaging using multi-slice multi-echo scanning sequence. Results showed that $\mathrm{T} 2$ relaxation time of the hippocampal region of interest in was significantly shorter in diabetic rats that in control group rats $(P=0.000$ Fig. $2 B)$. Thus, cognitive dysfunction occurred in diabetic rats. Particularly, the hippocampus was severely impaired as evidenced by iron deposition in hippocampal cells. 
Measurement of $\mathrm{Fe}^{2+}, \mathrm{MDA}$ and 4-HNE levels in the hippocampus of rats revealed that they were significantly higher in diabetic rats than in control group rats $(P=0.22, P=0.00, P=0.00$,Fig. $3 A, B, C)$. Electron microscopy showed that mitochondria in the hippocampal CA1 area of type 1 diabetic rats was significantly smaller while the sputum was shorter and diminished than in rats of control group, $(p=0.000$, Fig. 3D). Nissl's staining assay confirmed that Nissl stained areas was significantly lower in the hippocampal CA1 area of diabetic rats relative to rats of the control group (Fig. 3E). These indicated iron depositing in hippocampus which promoted ferroptosis in diabetic condition.

\section{Changes in mRNA/LncRNA expression profiles of hippocampus of type 1 diabetic rats}

To identify the key genes related to ferroptosis in hippocampus of diabetic rats, microarray analysis was conducted based on Rat LncRNA Microarray v2.0 comprising 24,626 protein coding mRNAs and 13,611 IncRNAs. A total of 201 dysregulated mRNAs and 101 IncRNAs (Supplementary Table S1 and S2) were found in hippocampal tissues of diabetic rats (Fig. 4A). In the pathway enrichment analyses, we found that mRNAs found to be differentially expressed were enriched in 'AGE-RAGE signaling pathway in diabetic complications', 'glycerophospholipid metabolism', 'Cell cycle', 'Mineral absorption', and 'Ferroptosis', (Fig. 4B). Among ferroptosis signaling pathways, Slc40a1 (Ferroportin) the main gene, was downregulated in diabetic rats (Fig. $4 \mathrm{C}$ ). These results suggested that ferroptosis was associated with diabetic cognitive dysfunction and Slc40a1 was the key gene that enhanced ferroptosis.

\section{Slc40a1 was significantly down-regulated in the hippocampus of type 1 diabetic rats}

The qRCR method was used to verify results of mRNA/IncRNA chip screening (The sequences of primers for PCR in Supplementary Table S3). SLC40A1, Atf3, LOC102554280, and LOC102551779 in the diabetic group were markedly lower (Fig. $5 A, P=0.03, P=0.000, P=0.018, P=0.034$ ) whereas Sult1a1, Heph, LOC103693580, LOC102549320 were markedly higher than in control group rats (Fig. 5A, $P=0.001, P$ $=0.001, P=0.001 P=0.004, P=0.002$ ). Good agreement was identified between the test results and the IncRNA/mRNA chip results, indicating that the results of mRNA/IncRNA chip were reliable. Western blotting assay confirmed that the expression of Slc40a1 was lower in hippocampal tissues of diabetic rats relative to control group rats (Fig. $5 \mathrm{~B}, \mathrm{p}=0.002$ )

\section{Discussion}

The development of cognitive dysfunction during diabetes is often latent and not easily recognized. Diabetes accelerates brain aging and promotes the occurrence of dementia [17]. Therefore, it is necessary to develop preventive measures to suppress cognitive impairment in diabetic conditions. Of note, diabetes is linked with cognitive impairment, especially type 2 diabetes, through multiple coexisting diseases (such as obesity, hypertension, hyperlipidemia). These coexisting diseases are independently associated with cognitive deficits [18]. To avoid interference caused by these underlying conditions, type 1 diabetes model was established in this study in rats. The pathogenesis of diabetes cognitive impairment includes impaired insulin signaling pathways, toxic effects of hyperglycemia, oxidative 
stress, vascular factors, and genetic factors [19]. Currently, its pathogenesis is not fully defined. Following the recent classification of ferroptosis as an independent type of cell death, several studies have been designed to define its mechanisms [20-22]. Evidence exists confirming that ferroptosis occurs in brain tissues given that these tissues contain the highest proportion of polyunsaturated fatty acids of all body tissues. For this reason, they have high lipid peroxidation levels [23]. Other studies have shown that ferroptosis influence the development of many neurologic diseases, such as Parkinson's disease, Alzheimer's disease, and stroke [24]. Against this background, we speculate that ferroptosis may drive the cognitive impairment in diabetic conditions. Currently, the link between diabetic cognitive dysfunction and ferroptosis is blurry.

A rat model of type 1 diabetic rat was created using streptozotocin (STZ). Cognitive function of diabetic rats was tested using a water maze experiment. Compared with normal group rats, diabetic rats showed a significantly longer escape latency in the positioning and navigation experiment, and spent shorter time in the target quadrant in the space exploration experiment. This indicates that their learning and memory ability was significantly reduced. In the subsequent experiment, we employed the rs-fMRI technology to assess the cognitive decline of type 1 diabetic rats by observing the brain structure and function change. Results showed that ALFF signals in bilateral hippocampus and anterior cingulate cortex were significantly reduced. The rs-fMRI technology which employs blood oxygenation level-dependent (BOLD) signals to quantify spontaneous brain activity is often exploited in neuroscience research [25]. In the application of this technology, the amplitude of low-frequency fluctuation (ALFF) [26] reflects the regional intensity of spontaneous fluctuations in the BOLD signal, which reveals the spontaneous neural activity in certain physiological states and brain regions. Thus, ALFF is an ideal measure that can reveal the brain activity [27]. A decrease in ALFF indicates that the spontaneous activity of neurons in the corresponding brain area is weakened and structure function is impaired. In contrast, increase in ALFF indicates enhanced brain activity $[28,29]$. In our study, ALFF signals in the hippocampus and anterior cingulate cortex of type 1 diabetic rats were reduced, indicating that these central neurons related to learning and memory activity were weakened. Based on this, it was confirmed that type 1 diabetes compromised cognition functions, which is broadly consistent with clinical studies [30]. In addition, the multi-layer multi-echo scanning sequence T2map-MSME was used to detect the T2 relaxation time of the hippocampus. This test showed that the relaxation time of the hippocampus of diabetic rats was shorter compared with rats in the normal group. T2 relaxation time reflects the brain iron content to a certain extent, and the two are negatively correlated. That is, the shorter the relaxation time, the higher the brain iron content [31, 32]. In this study, iron content in the hippocampus was measured with colorimetric method. Results revealed that the iron content of diabetic rats was higher relative to that of rats in normal group. Thus, our findings confirm that cognitive decline in type 1 diabetic rats is accompanied by iron deposition in hippocampal tissues.

Ferroptosis is recently defined type of cell death, often reflected by iron-dependent lipid peroxidation causing cell death and oxidative stress [33]. Several products are produced during lipid peroxidation. The process of peroxidation is initiated by lipid hydro-peroxides (LOOHs). Other secondary substrates that drive peroxidation include aldehydes. Notably, 4-hydroxynonenal (4-HNE) and malondialdehyde (MDA) 
have been well-studied [34]. Lipid peroxidation results in production of MDA which has mutagenic effects as it reacts with DNA and primary amines on proteins forming crosslinked adducts [35]. Studies have implicated electrophile 4-HNE in cell proliferation and cell cycle progression where it causes cytotoxicity and suppresses gene expression. In this way, it accelerates disease development [34]. In this study, levels of 4-HNE and MDA in hippocampal tissues of diabetic rats higher than in normal rats. This indicates that diabetes is associated with lipid peroxidation. Further, mitochondrial morphology in the hippocampal $\mathrm{CA}_{1}$ area of the rat was examined with a transmission electron microscope. Results showed that compared with normal rats, the mitochondria in the hippocampus of diabetic rats were shrunken, lacked ridges, and the mitochondrial membrane thickened. All this mitochondria characteristics were similar to those reported for ferroptosis previously [2]. Nissl body assay was employed to detect the degree of neuronal damage. Damaged neurons reduce the Nissl body [36]. In our study, compared with the control group, a significant reduction in Nissl was observed in the hippocampal CA1 area in diabetic rats. Therefore, we inferred that ferroptosis occurred in the hippocampus of diabetic rats.

We further used gene chip technique to identify key genes related to ferroptosis in hippocampal tissues in diabetic rats. The high-throughput-screening revealed 101 IncRNAs and 201 abnormally expressed mRNAs in the hippocampus of diabetic rats. In the pathway enrichment analyses, differentially expressed mRNAs were enriched in 'AGE-RAGE signaling pathway in diabetic complications' 'glycerophospholipid metabolism', 'Cell cycle', 'Mineral absorption', and 'Ferroptosis'. Our results demonstrated that ferroptosis was associated with diabetic cognitive dysfunction. Among ferroptosis signaling pathways, SLC40A1 the key gene, was significantly lower in diabetic rat hippocampus relative to control rats.

SLC40A1 encodes a ferroportin (FPN). FPN is the only iron export protein on the cell membrane that maintains iron homeostasis $[37,38]$. Downregulation of SLC40A1 compromises intracellular iron output, causing iron overload. Some scholars have found that knockdown FPN on the membrane of neuroblastoma cells can accelerate ferroptosis in cells.[39] Other reports show that SLC40A1 is associated with Alzheimer's disease [40-42]. These findings suggest that ferroptosis induced by downregulation of SLC40A1 may be an important pathogenic mechanism of cognitive dysfunction.

Intracellular iron content is tightly regulated in the body through iron transport-related proteins on the cell membrane, including divalent metal ion transporter proteins (Divalent metal ion transporter 1, DMT1), Ceruloplasmin (CP), Transferrin receptor (TFR), and Ferroportin (FPN). Among these membrane proteins, FPN is only the membrane transferrin that transports iron out of the cell. Therefore, we speculate that SLC40A1 may be a key pathogenic gene driving iron deposition and ferroptosis in the hippocampus of type 1 diabetic rats. In future research, the SLC40A1 adeno-associated virus (AAV) vector should be constructed to facilitate further inquiry into the role of SLC40A1 in cognitive dysfunction in type 1 diabetes.

In summary, our results indicate that down-regulation of SLC40A1 causes iron deposition and ferroptosis in the brain, and the subsequent cognitive dysfunction in type 1 diabetes.

\section{Declarations}

Page 10/19 
Ethics approval and consent to participate:Animal experiment protocols in this study were in accordance with guidelines established by the China's Council on Animal Care and approved by the Shanxi Medical University Animal Care Committee.

Consent for publication:Not applicable

Availability of data and materials:Please contact author for data requests.

Funding:No funding support

Competing interests:

The authors declare that they have no competing interests.

\section{Authors' contributions:}

CZ conceived and designed the experiments; LJH performed the experiments; JM, LPS and YNG analyzed and contributed reagents/materials/analysis tools; YLL, and YRY wrote the paper. All authors reviewed the manuscript.

Acknowledgements:Not applicable

\section{References}

[1] S. Chatterjee, K. Khunti, M.J. Davies, Type 2 diabetes, Lancet 389(10085) (2017) 2239-2251.

[2] R.J. McCrimmon, C.M. Ryan, B.M. Frier, Diabetes and cognitive dysfunction, Lancet 379(9833) (2012) 2291-9.

[3] C. Moucheraud, C. Lenz, M. Latkovic, V.J. Wirtz, The costs of diabetes treatment in low- and middleincome countries: a systematic review, BMJ Glob Health 4(1) (2019) e001258.

[4] H.G. Kim, Cognitive dysfunctions in individuals with diabetes mellitus, Yeungnam Univ J Med 36(3) (2019) 183-191.

[5] C.T. Kodl, E.R. Seaquist, Cognitive dysfunction and diabetes mellitus, Endocr Rev 29(4) (2008) 494511.

[6] S.J. Dixon, K.M. Lemberg, M.R. Lamprecht, R. Skouta, E.M. Zaitsev, C.E. Gleason, D.N. Patel, A.J. Bauer, A.M. Cantley, W.S. Yang, B. Morrison, 3rd, B.R. Stockwell, Ferroptosis: an iron-dependent form of nonapoptotic cell death, Cell 149(5) (2012) 1060-72.

[7] B.R. Stockwell, J.P. Friedmann Angeli, H. Bayir, A.I. Bush, M. Conrad, S.J. Dixon, S. Fulda, S. Gascon, S.K. Hatzios, V.E. Kagan, K. Noel, X. Jiang, A. Linkermann, M.E. Murphy, M. Overholtzer, A. Oyagi, G.C. Pagnussat, J. Park, Q. Ran, C.S. Rosenfeld, K. Salnikow, D. Tang, F.M. Torti, S.V. Torti, S. Toyokuni, K.A. 
Woerpel, D.D. Zhang, Ferroptosis: A Regulated Cell Death Nexus Linking Metabolism, Redox Biology, and Disease, Cell 171(2) (2017) 273-285.

[8] R.P. Abrams, W.L. Carroll, K.A. Woerpel, Five-Membered Ring Peroxide Selectively Initiates Ferroptosis in Cancer Cells, ACS chemical biology 11(5) (2016) 1305-12.

[9] R. Sheelakumari, C. Kesavadas, T. Varghese, R.M. Sreedharan, B. Thomas, J. Verghese, P.S. Mathuranath, Assessment of Iron Deposition in the Brain in Frontotemporal Dementia and Its Correlation with Behavioral Traits, AJNR. American journal of neuroradiology 38(10) (2017) 1953-1958.

[10] A.A. Belaidi, A.I. Bush, Iron neurochemistry in Alzheimer's disease and Parkinson's disease: targets for therapeutics, J Neurochem 139 Suppl 1 (2016) 179-197.

[11] L. Magtanong, S.J. Dixon, Ferroptosis and Brain Injury, Developmental neuroscience (2019) 1-14.

[12] G. Morris, M. Berk, A.F. Carvalho, M. Maes, A.J. Walker, B.K. Puri, Why should neuroscientists worry about iron? The emerging role of ferroptosis in the pathophysiology of neuroprogressive diseases, Behavioural brain research 341 (2018) 154-175.

[13] N. Yan, J. Zhang, Iron Metabolism, Ferroptosis, and the Links With Alzheimer's Disease, Front Neurosci 13 (2019) 1443.

[14] Y. Mi, X. Gao, H. Xu, Y. Cui, Y. Zhang, X. Gou, The Emerging Roles of Ferroptosis in Huntington's Disease, Neuromolecular Med 21(2) (2019) 110-119.

[15] S. Ayton, P. Lei, D.J. Hare, J.A. Duce, J.L. George, P.A. Adlard, C. McLean, J.T. Rogers, R.A. Cherny, D.I. Finkelstein, A.I. Bush, Parkinson's disease iron deposition caused by nitric oxide-induced loss of betaamyloid precursor protein, J Neurosci 35(8) (2015) 3591-7.

[16] Y. Ohyagi, K. Miyoshi, N. Nakamura, Therapeutic Strategies for Alzheimer's Disease in the View of Diabetes Mellitus, Adv Exp Med Biol 1128 (2019) 227-248.

[17] G.J. Biessels, L.P. van der Heide, A. Kamal, R.L. Bleys, W.H. Gispen, Ageing and diabetes: implications for brain function, European journal of pharmacology 441(1-2) (2002) 1-14.

[18] C.M. Embury, A.I. Wiesman, A.L. Proskovec, E. Heinrichs-Graham, T.J. McDermott, G.H. Lord, K.L. Brau, A.T. Drincic, C.V. Desouza, T.W. Wilson, Altered Brain Dynamics in Patients With Type 1 Diabetes During Working Memory Processing, Diabetes 67(6) (2018) 1140-1148.

[19] G.J. Biessels, I.J. Deary, C.M. Ryan, Cognition and diabetes: a lifespan perspective, The Lancet. Neurology 7(2) (2008) 184-90.

[20] S.E. Wenzel, Y.Y. Tyurina, J. Zhao, C.M. St Croix, H.H. Dar, G. Mao, V.A. Tyurin, T.S. Anthonymuthu, A.A. Kapralov, A.A. Amoscato, K. Mikulska-Ruminska, I.H. Shrivastava, E.M. Kenny, Q. Yang, J.C. Rosenbaum, 
L.J. Sparvero, D.R. Emlet, X. Wen, Y. Minami, F. Qu, S.C. Watkins, T.R. Holman, A.P. VanDemark, J.A. Kellum, I. Bahar, H. Bayir, V.E. Kagan, PEBP1 Wardens Ferroptosis by Enabling Lipoxygenase Generation of Lipid Death Signals, Cell 171(3) (2017) 628-641.e26.

[21] J.Y. Cao, S.J. Dixon, Mechanisms of ferroptosis, Cell Mol Life Sci 73(11-12) (2016) 2195-209.

[22] J.P.F. Angeli, R. Shah, D.A. Pratt, M. Conrad, Ferroptosis Inhibition: Mechanisms and Opportunities, Trends Pharmacol Sci 38(5) (2017) 489-498.

[23] R.P. Bazinet, S. Laye, Polyunsaturated fatty acids and their metabolites in brain function and disease, Nat Rev Neurosci 15(12) (2014) 771-85.

[24] A. Weiland, Y. Wang, W. Wu, X. Lan, X. Han, Q. Li, J. Wang, Ferroptosis and Its Role in Diverse Brain Diseases, Mol Neurobiol 56(7) (2019) 4880-4893.

[25] K.V. Haak, A.F. Marquand, C.F. Beckmann, Connectopic mapping with resting-state fMRI, Neuroimage 170 (2018) 83-94.

[26] Y.F. Zang, Y. He, C.Z. Zhu, Q.J. Cao, M.Q. Sui, M. Liang, L.X. Tian, T.Z. Jiang, Y.F. Wang, Altered baseline brain activity in children with ADHD revealed by resting-state functional MRI, Brain Dev 29(2) (2007) 83-91.

[27] V. Kiviniemi, J.H. Kantola, J. Jauhiainen, O. Tervonen, Comparison of methods for detecting nondeterministic BOLD fluctuation in fMRI, Magn Reson Imaging 22(2) (2004) 197-203.

[28] L.X. Yuan, J.B. Wang, N. Zhao, Y.Y. Li, Y. Ma, D.Q. Liu, H.J. He, J.H. Zhong, Y.F. Zang, Intra- and Interscanner Reliability of Scaled Subprofile Model of Principal Component Analysis on ALFF in Resting-State fMRI Under Eyes Open and Closed Conditions, Frontiers in neuroscience 12 (2018) 311.

[29] P. Wang, J. Yang, Z. Yin, J. Duan, R. Zhang, J. Sun, Y. Xu, L. Liu, X. Chen, H. Li, J. Kang, Y. Zhu, X. Deng, M. Chang, S. Wei, Y. Zhou, X. Jiang, F. Wang, Y. Tang, Amplitude of low-frequency fluctuation (ALFF) may be associated with cognitive impairment in schizophrenia: a correlation study, BMC Psychiatry 19(1) (2019) 30.

[30] Q. Yang, L. Zhou, C. Liu, D. Liu, Y. Zhang, C. Li, Y. Shang, X. Wei, C. Li, J. Wang, Brain iron deposition in type 2 diabetes mellitus with and without mild cognitive impairment-an in vivo susceptibility mapping study, Brain imaging and behavior 12(5) (2018) 1479-1487.

[31] K.M. Hasan, I.S. Walimuni, L.A. Kramer, P.A. Narayana, Human brain iron mapping using atlas-based T2 relaxometry, Magn Reson Med 67(3) (2012) 731-739.

[32] E.M. Haacke, N.Y.C. Cheng, M.J. House, Q. Liu, J. Neelavalli, R.J. Ogg, A. Khan, M. Ayaz, W. Kirsch, A. Obenaus, Imaging iron stores in the brain using magnetic resonance imaging, Magnetic resonance imaging 23(1) (2005) 1-25. 
[33] S. Doll, M. Conrad, Iron and ferroptosis: A still ill-defined liaison, IUBMB Life 69(6) (2017) 423-434.

[34] A. Ayala, M.F. Munoz, S. Arguelles, Lipid peroxidation: production, metabolism, and signaling mechanisms of malondialdehyde and 4-hydroxy-2-nonenal, Oxid Med Cell Longev 2014 (2014) 360438.

[35] N. Zarkovic, A. Cipak, M. Jaganjac, S. Borovic, K. Zarkovic, Pathophysiological relevance of aldehydic protein modifications, J Proteomics 92 (2013) 239-47.

[36] S.M. Chiroma, M.T. Hidayat Baharuldin, C.N. Mat Taib, Z. Amom, S. Jagadeesan, M.I. Adenan, M.A. Mohd Moklas, Protective effect of Centella asiatica against D-galactose and aluminium chloride induced rats: Behavioral and ultrastructural approaches, Biomed Pharmacother 109 (2019) 853-864.

[37] M. Theurl, D. Song, E. Clark, J. Sterling, S. Grieco, S. Altamura, B. Galy, M. Hentze, M.U. Muckenthaler, J.L. Dunaief, Mice with hepcidin-resistant ferroportin accumulate iron in the retina, FASEB journal : official publication of the Federation of American Societies for Experimental Biology 30(2) (2016) 813-23.

[38] S. Lakhal-Littleton, M. Wolna, C.A. Carr, J.J. Miller, H.C. Christian, V. Ball, A. Santos, R. Diaz, D. Biggs, R. Stillion, P. Holdship, F. Larner, D.J. Tyler, K. Clarke, B. Davies, P.A. Robbins, Cardiac ferroportin regulates cellular iron homeostasis and is important for cardiac function, Proceedings of the National Academy of Sciences of the United States of America 112(10) (2015) 3164-9.

[39] N. Geng, B.J. Shi, S.L. Li, Z.Y. Zhong, Y.C. Li, W.L. Xua, H. Zhou, J.H. Cai, Knockdown of ferroportin accelerates erastin-induced ferroptosis in neuroblastoma cells, European review for medical and pharmacological sciences 22(12) (2018) 3826-3836.

[40] F. Du, Z.M. Qian, Q. Luo, W.H. Yung, Y. Ke, Hepcidin Suppresses Brain Iron Accumulation by Downregulating Iron Transport Proteins in Iron-Overloaded Rats, Molecular neurobiology 52(1) (2015) 101-14.

[41] R.C. McCarthy, Y.H. Park, D.J. Kosman, sAPP modulates iron efflux from brain microvascular endothelial cells by stabilizing the ferrous iron exporter ferroportin, EMBO reports 15(7) (2014) 809-15.

[42] A.A. Raha, R.A. Vaishnav, R.P. Friedland, A. Bomford, R. Raha-Chowdhury, The systemic ironregulatory proteins hepcidin and ferroportin are reduced in the brain in Alzheimer's disease, Acta neuropathologica communications 1 (2013) 55.

\section{Figures}




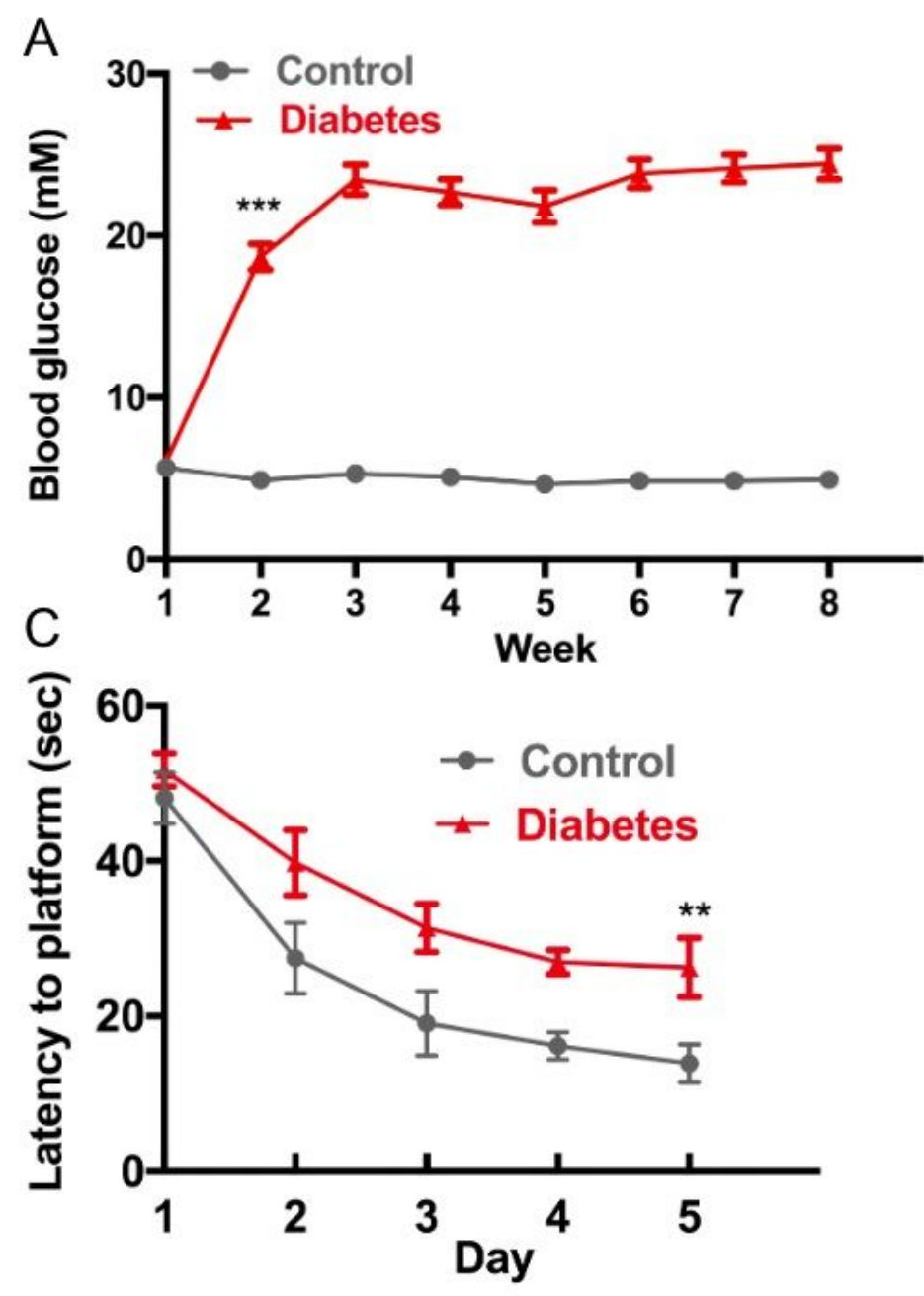

B

$E$

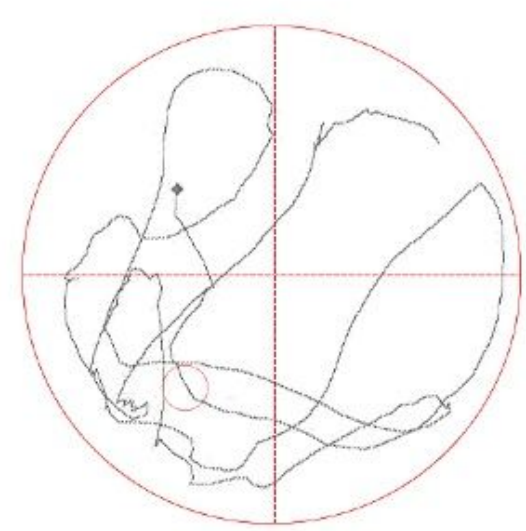

$\mathrm{F}$

Control
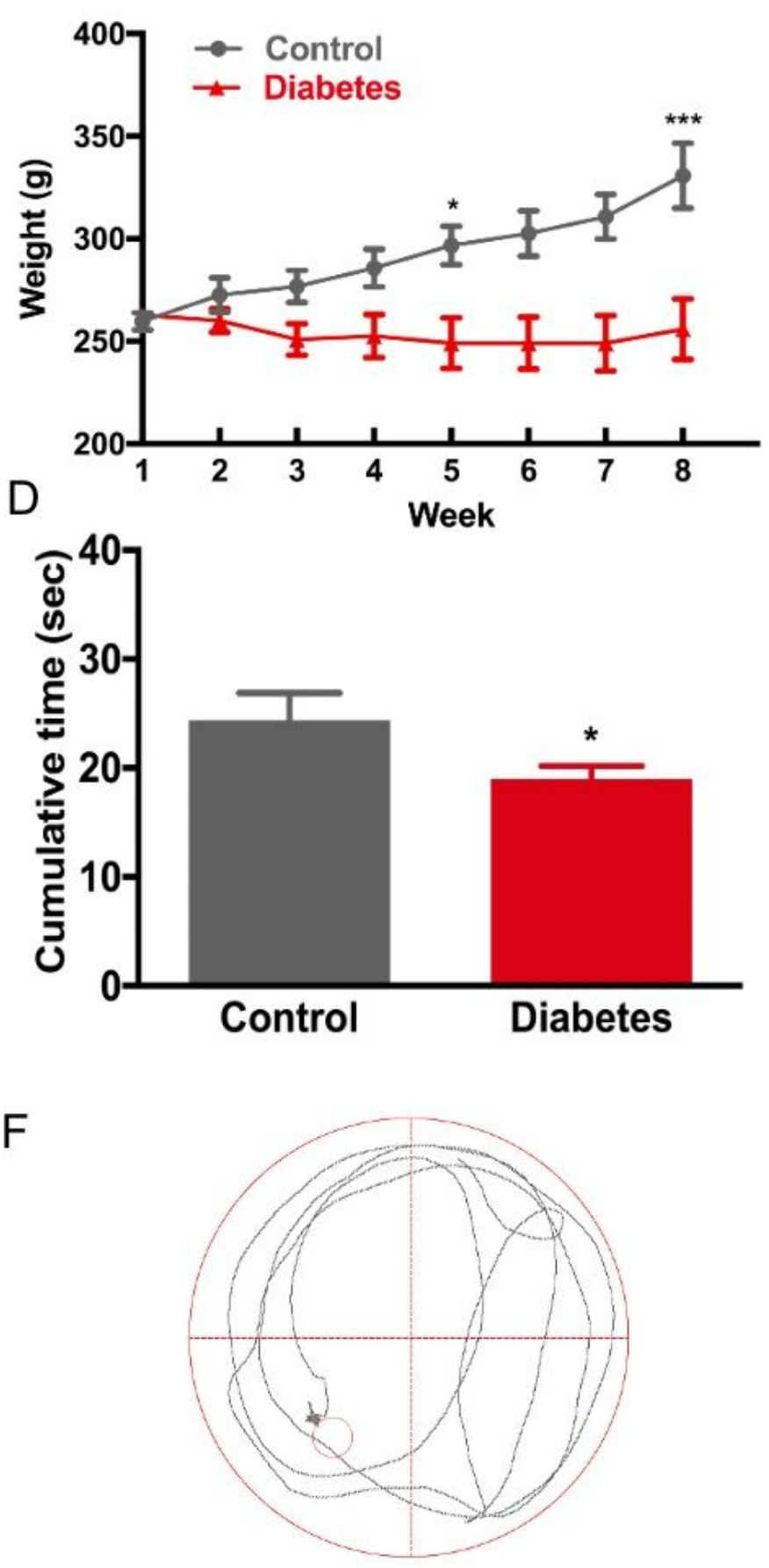

Diabetes

Figure 1

Cognitive function, body weight, and blood glucose level in diabetic rats. A, the fasting blood glucose level in the groups. B, body weight in the two groups. $C$, the time spent to find the hidden platform in the hidden platform test. $\mathrm{D}$, the cumulative time spent in the target quadrant during the probe trial test. $\mathrm{E}$ and $F$, swim traces of control and diabetes rats in probe trials. ${ }^{*}$, ${ }^{\star}$, and ${ }^{* \star \star}$ notes $p<0.05, p<0.01$, and $p<$ 0.001 vs. control, respectively. 

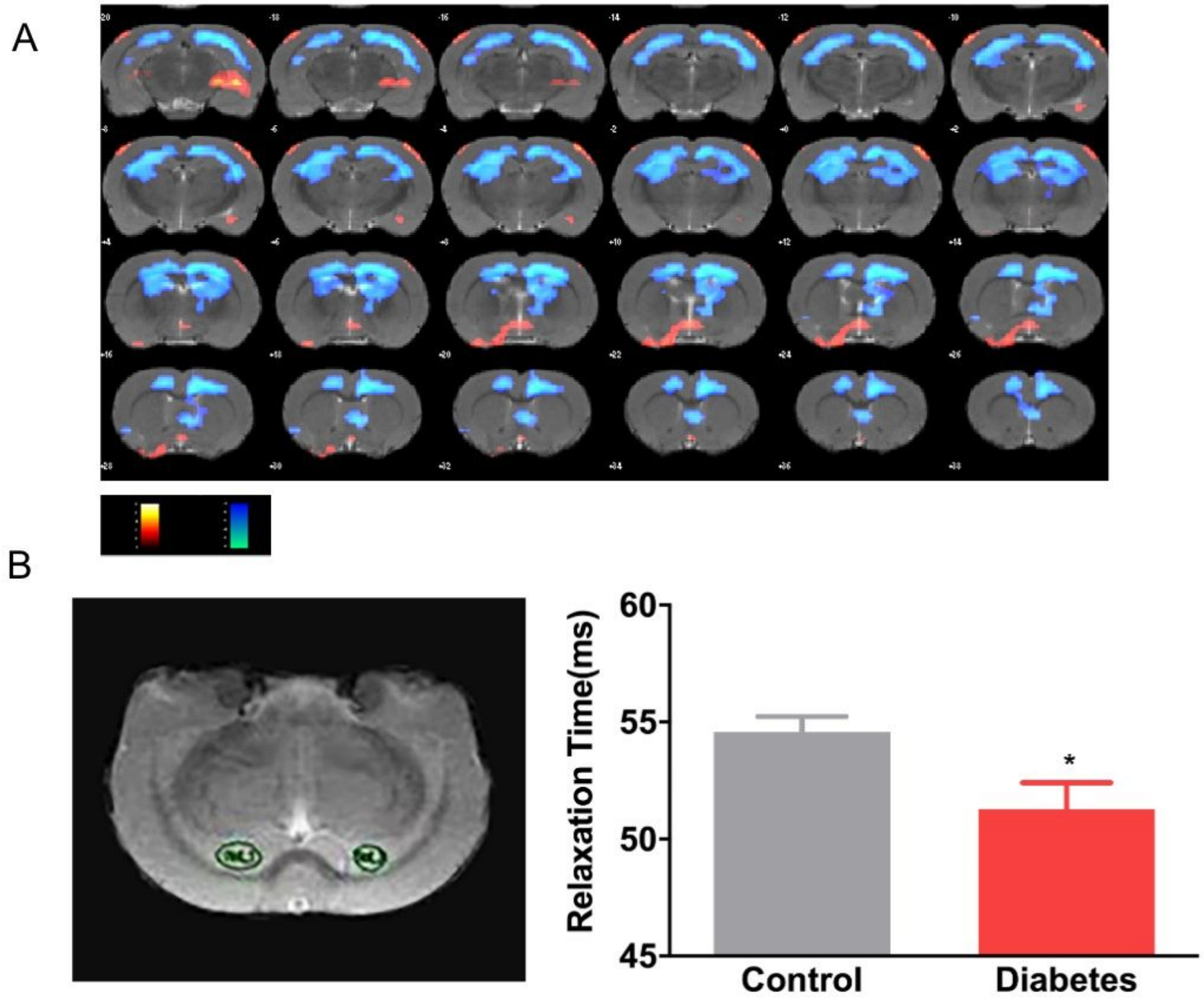

Figure 2

ALFF value and T2 relaxation time of brain in diabetic rats. A, the changes of ALFF value of different areas of brain, red represents ALFF increase areas of the brain, blue represents ALFF lower areas of the brain. $B, T 2$ relaxation time of hippocampus. ${ }^{*}$, notes $p<0.05$, vs. control. 
A

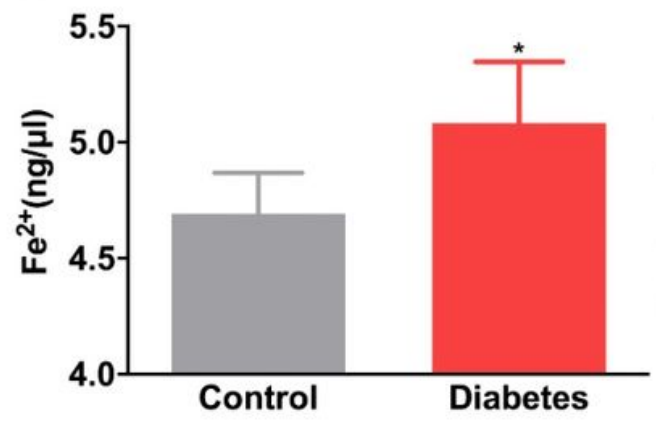

D

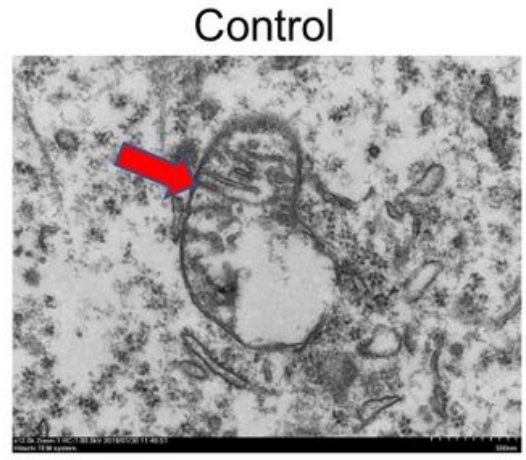

E

Control
B

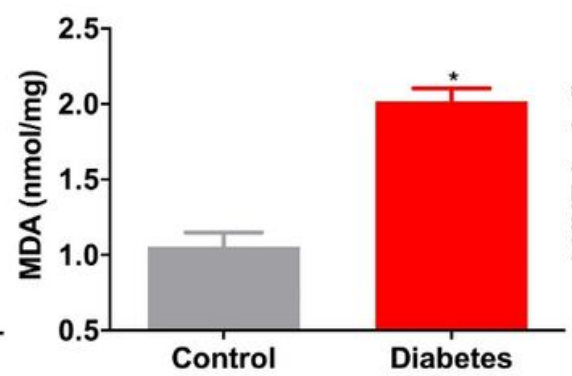

Diabetes
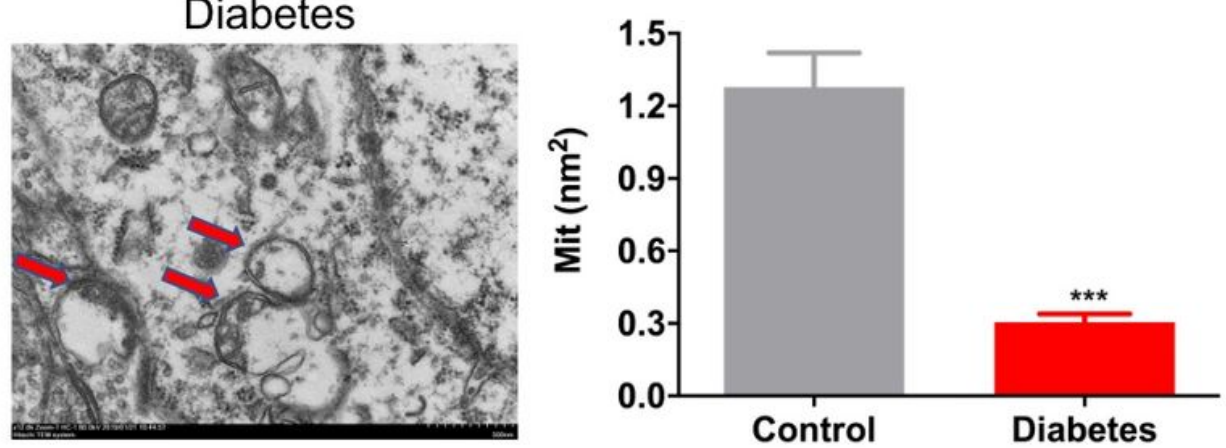

Control
C

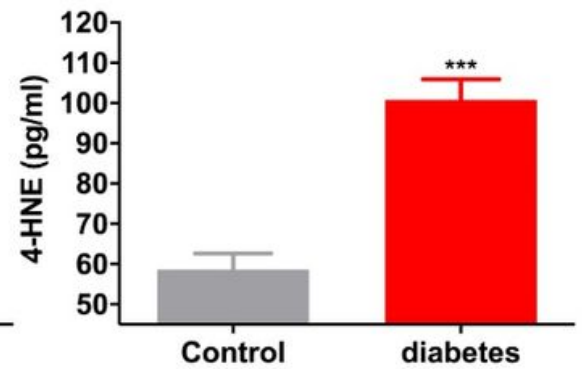

Diabetes

Diabetes

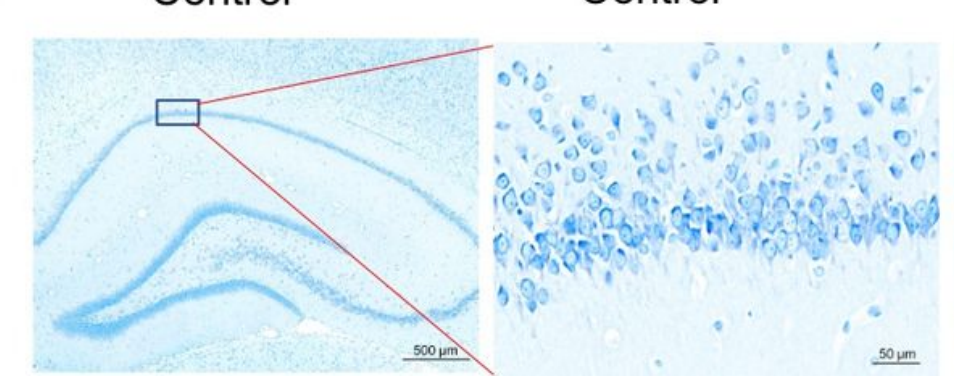

\section{Figure 3}

Fe2+, MDA, 4-HNE level in hippocampus, and Histopathological examinations for hippocampus. A, B and $\mathrm{C}$ represented Fe2+, MDA, 4-HNE level in hippocampus of diabetic and control rats respectively. D, represented images of transmission electron microscopy, red arrow pointing mitochondrial morphology of hippocampal CA1 region (Magnification $\times 12000$ ), the area of mitochondrial was counted. E, Nissl's staining imaging results (Magnification $\times 20$ and Magnification $\times 160$ ). ${ }^{*}$, and ${ }^{* *}$ notes $p<0.05$, and $p<$ 0.001 vs. control, respectively. 
A
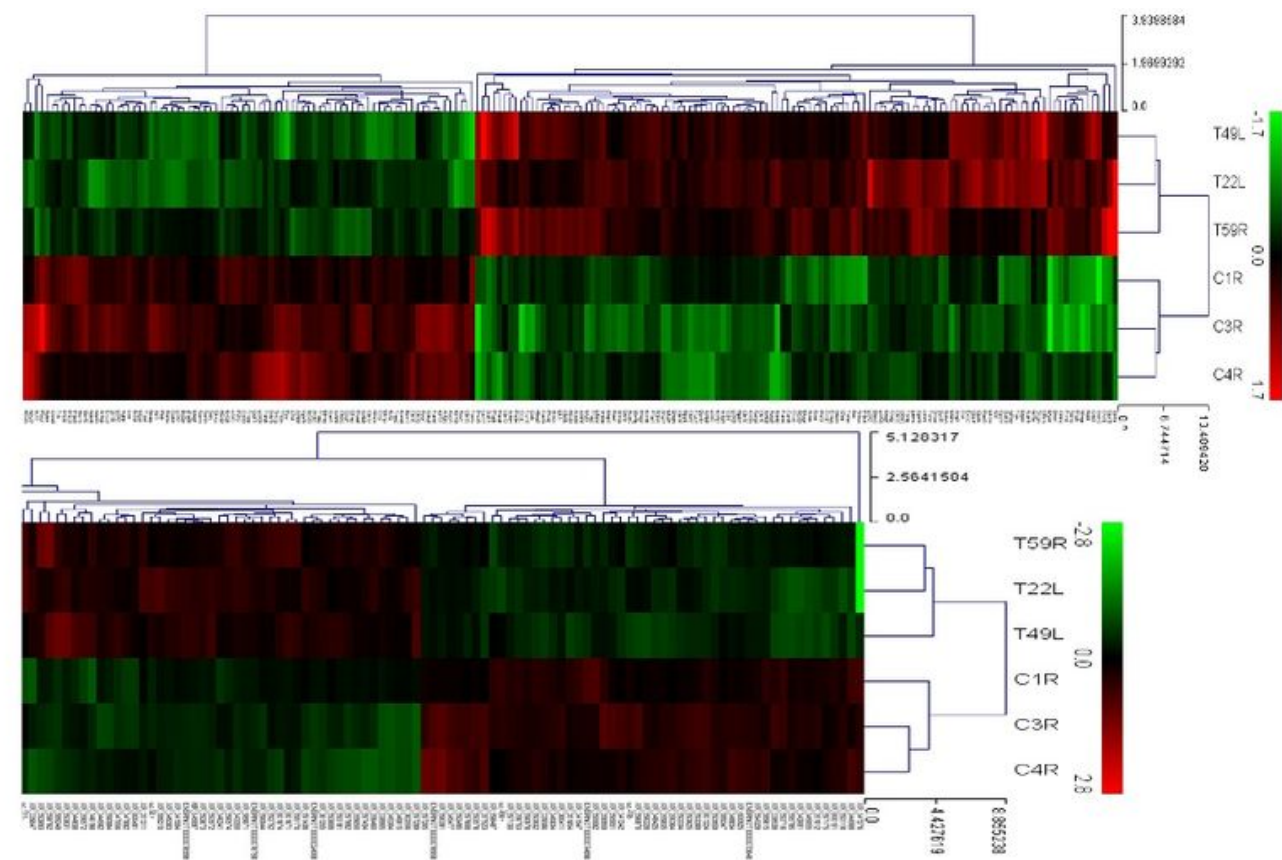

B

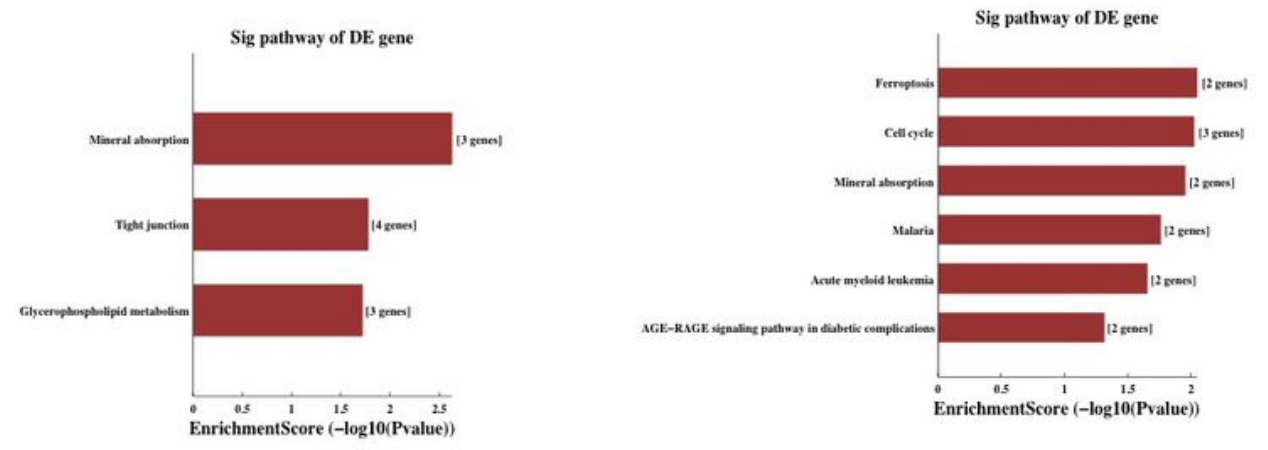

C

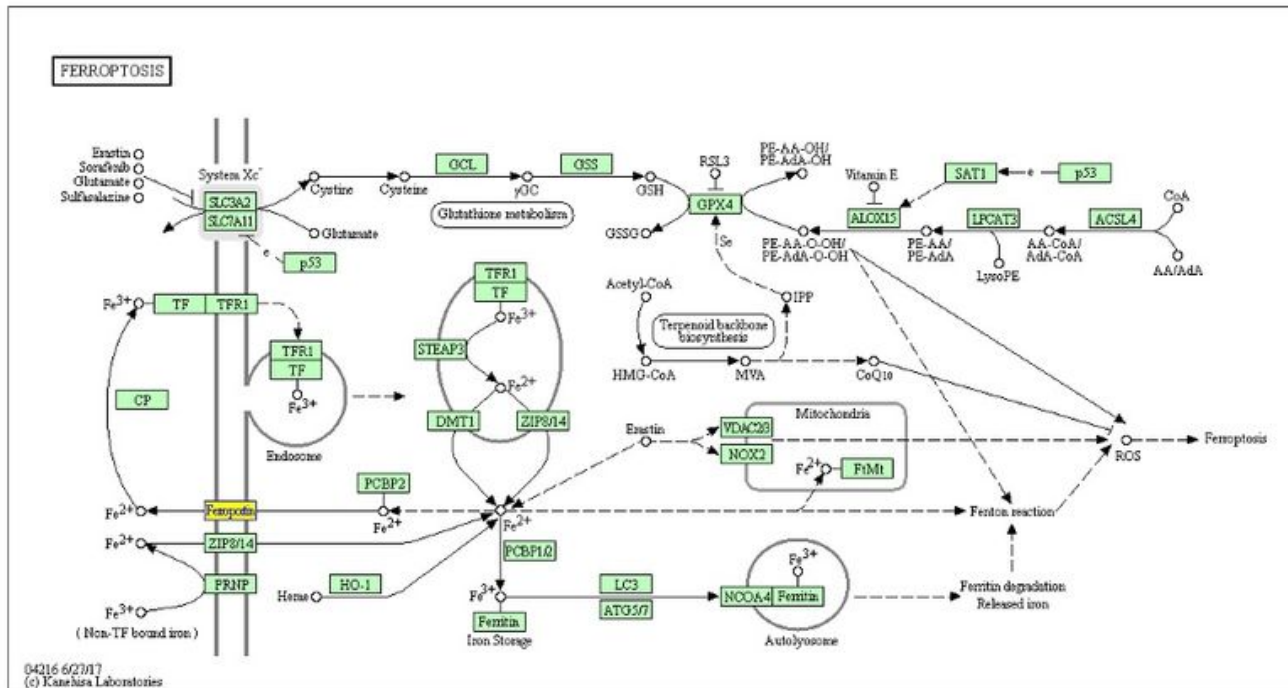

\section{Figure 4}

Identification and annotation of differentially expressed mRNAs and IncRNAs in diabetic rats. A, the cluster of differentially expressed mRNAs and IncRNAs. B, the significant pathways of the mRNAs. C,The gene marked yellow represents downregulated. 
B

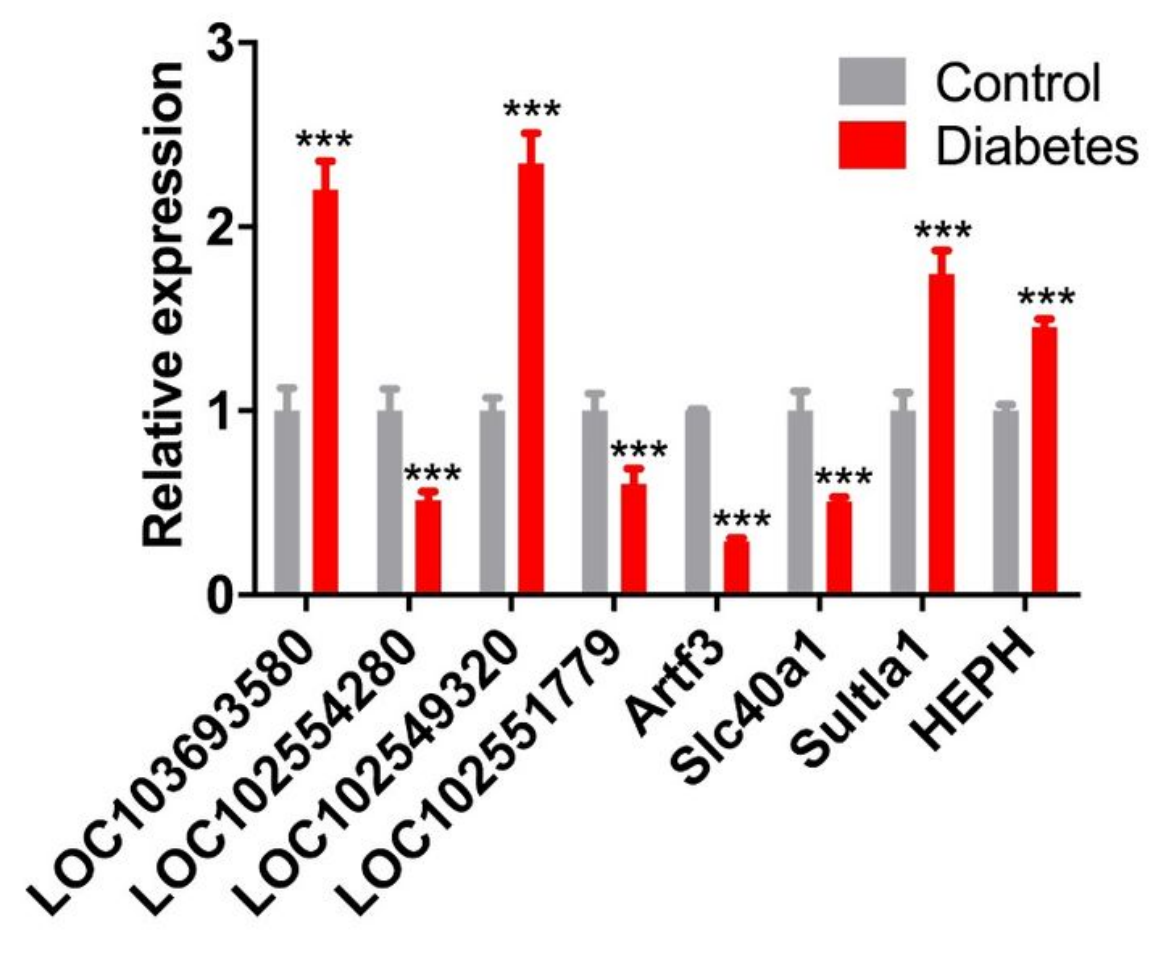

Slc40a1
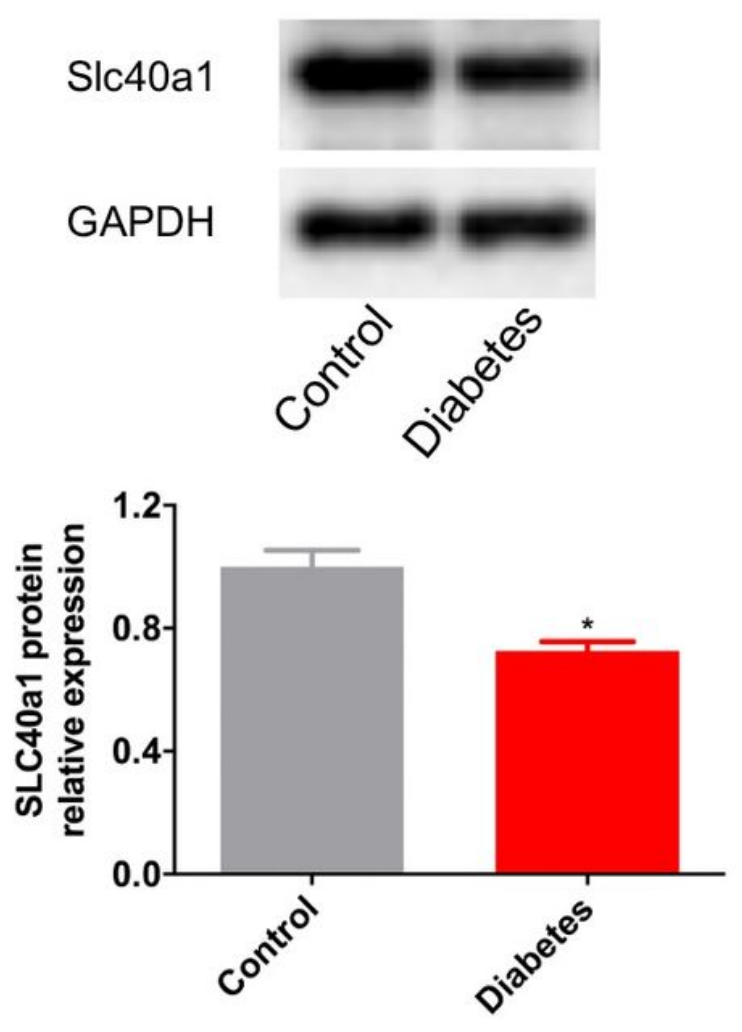

Figure 5

qPCR and Western blot analysis. A, the relative expression level of 8 significantly expressed RNAs in diabetic rats. B, fold change of Slc40a1 protein. ${ }^{*}$ and ${ }^{* * *}$ notes $p<0.05$ and $p<0.001$ vs. control, respectively.

\section{Supplementary Files}

This is a list of supplementary files associated with this preprint. Click to download.

- SupplementaryTable2.xls

- SupplementaryFigure1.tiff

- SupplementaryTable3.xlsx

- SupplementaryFigure3.pdf

- SupplementaryFigure2.tiff

- SupplementaryFigure4.tiff

- SupplementaryFigure5.tiff

- SupplementaryFigure6.tiff 